 \\ UNIVERSITY OF \\ TECHNOLOGY
}

\section{Bubble impingement in the presence of a solid particle}

\section{Citation for published version (APA):}

Sannino, A., Esposito, A., Villone, M. M., Hulsen, M. A., \& D'Avino, G. (2018). Bubble impingement in the presence of a solid particle: a computational study. Computers \& Fluids, 170, 349-356.

https://doi.org/10.1016/j.compfluid.2018.05.009

DOI:

10.1016/j.compfluid.2018.05.009

Document status and date:

Published: 15/07/2018

\section{Document Version:}

Accepted manuscript including changes made at the peer-review stage

\section{Please check the document version of this publication:}

- A submitted manuscript is the version of the article upon submission and before peer-review. There can be important differences between the submitted version and the official published version of record. People interested in the research are advised to contact the author for the final version of the publication, or visit the $\mathrm{DOI}$ to the publisher's website.

- The final author version and the galley proof are versions of the publication after peer review.

- The final published version features the final layout of the paper including the volume, issue and page numbers.

Link to publication

\section{General rights}

Copyright and moral rights for the publications made accessible in the public portal are retained by the authors and/or other copyright owners and it is a condition of accessing publications that users recognise and abide by the legal requirements associated with these rights.

- Users may download and print one copy of any publication from the public portal for the purpose of private study or research.

- You may not further distribute the material or use it for any profit-making activity or commercial gain

- You may freely distribute the URL identifying the publication in the public portal.

If the publication is distributed under the terms of Article 25fa of the Dutch Copyright Act, indicated by the "Taverne" license above, please follow below link for the End User Agreement:

www.tue.nl/taverne

Take down policy

If you believe that this document breaches copyright please contact us at:

openaccess@tue.nl

providing details and we will investigate your claim. 


\title{
Bubble impingement in the presence of a solid particle: a computational study
}

\author{
Andrea Sannino, Alessandro Esposito, Massimiliano M. Villone* \\ Dipartimento di Ingegneria Chimica, dei Materiali e della Produzione Industriale, Università di Napoli \\ Federico II, P.le Tecchio 80, 80125 Napoli, Italy \\ Martien A. Hulsen \\ Department of Mechanical Engineering, Eindhoven University of Technology, PO Box 513, 5600 MB \\ Eindhoven, The Netherlands \\ Gaetano D'Avino \\ Dipartimento di Ingegneria Chimica, dei Materiali e della Produzione Industriale, Università di Napoli \\ Federico II, P.le Tecchio 80, 80125 Napoli, Italy
}

\begin{abstract}
In this work, we numerically investigate the dynamics of the growth and impingement of two gas bubbles in a Newtonian liquid in the presence of a rigid spherical particle. The computational analysis is carried out through 3D Arbitrary Lagrangian Eulerian (ALE) Finite Element Method (FEM) simulations.

During their growth, as the bubbles start to 'feel' each other, they lose their spherical shape, with the side facing the other bubble becoming almost flat. In the liquid layer between the gas inclusions, an essentially biaxial extensional flow takes place. Depending on its initial position with respect to the bubbles, the solid particle can be 'captured' by the coalescing bubbles or 'escape' them. The effects of the physical and geometrical parameters of the system on such phenomenon are studied.
\end{abstract}

Keywords: Soft Matter, Foam, Bubble growth, Impingement, Solid particle, Direct Numerical Simulations

\footnotetext{
${ }^{*}$ Corresponding author. +390817682280

Email address: massimiliano.villone@unina.it (Massimiliano M. Villone)
} 


\section{Introduction}

Foams are ubiquitous in nature. Due to their mass and energy transport, acoustic and impact absorption, and catalytic properties, they are also of great interest in science and industrial applications ranging from construction to food, from packaging to biomedicine [1, 2].

The first investigations on the dynamics of gas bubbles in a liquid date back to one century ago, when Lord Rayleigh studied the pressure field in a liquid containing a collapsing bubble [3]. Some years later, Lamb considered a somewhat opposite problem, namely, the explosive growth of submarine bubbles [4]. Since then, foams have received a great interest in the literature: numerous theoretical, experimental, and numerical works deal with the formation, growth (or collapse), and coalescence of gas bubbles in liquid matrices.

When a gas is dispersed into a liquid, the presence of inhomogeneities or impurities can make it nucleate, thus forming little bubbles, which can grow because of gas diffusion through the liquid phase [5, 6]. Since the second half of the $20^{\text {th }}$ century, several models of the growth of a single gas bubble in a Newtonian liquid have been developed [7, 8]. Favelukis et al. developed an analytical prediction for spherical bubble growth in an infinite generalized Newtonian medium [9]. In its 'simple' Newtonian version, the model reads

$$
\begin{array}{r}
\frac{R}{R_{0}}=\frac{2 \sigma}{R_{0}\left(p_{\mathrm{b}}-p_{\infty}\right)}+ \\
+\left[1-\frac{2 \sigma}{R_{0}\left(p_{\mathrm{b}}-p_{\infty}\right)}\right] e^{\frac{p_{\mathrm{b}}-p_{\infty}}{4 \eta} t}
\end{array}
$$

with $R$ the bubble actual radius, $R_{0}$ the bubble initial radius, $\sigma$ the surface tension between the liquid and the gas, $p_{\mathrm{b}}$ the bubble pressure (supposed constant), $p_{\infty}$ the liquid pressure far from the growing bubble (constant as well), $\eta$ the liquid viscosity, and $t$ the time.

When numerous gas bubbles are dispersed in a liquid at distances much larger than their dimensions, the spherical growth models adequately describe their dynamics. When this is not the case, such models are still suitable for the description of the very early inflation stages, but fail to describe the growing dynamics when the distances among bubbles become comparable with their dimensions. Indeed, it is known that, when gas bubbles approach impingement, i.e., the thickness of the liquid layer separating them gets progressively thinner, they attain a non-spherical shape, as the sides facing other bubbles become almost flat. Hence, if a growing bubble is surrounded by other gas inclusions, it assumes a polyhedron shape [10, 11]. The evolution of the thickness, stress state, and deformation of the liquid film during bubble growth and impingement has received a great interest in the literature, especially with reference to polymer foams, and has been the subject of numerous experimental and numerical works over the last two decades [12, 13, 14, 6, 15, 16, 17].

When the liquid film separating growing bubbles thins below a critical thickness, a spontaneous rupture can happen [18]. In polymer foams, local flow-induced crystallization of the liquid can occur during bubble growth: the so-formed inhomogeneity can promote film rupture [19]. In any kind of liquid 
matrix, bubble walls may also break because of the presence of impurities like solid particles [20, 21, 22]. Bubble rupture, in turn, can open the way to liquid film retraction, whose extent and velocity depend on the interplay among inertial, surface, viscous, and elastic forces (in case the liquid phase is viscoelastic) [23, 24, 25, 26, 27]. The presence of solid objects suspended in the liquid matrix, then, can play a crucial role, since it can anticipate bubble rupture and affect the final morphology of the material [27]. Specifically, the presence of solid particles may generate high local stress fields between the particle and the surface of a close bubble, leading to the formation of a hole on its surface and, consequently, bubble rupture. However, despite the relevance of solid inclusions in foaming processes, bubble growth dynamics has been always studied assuming a pure and homogenous matrix liquid.

In this paper, we study the growth and impingement of gas bubbles in a Newtonian liquid in the presence of a solid particle through fully three-dimensional Direct Numerical Simulations (DNS). A model system consisting in a liquid matrix containing two bubbles inflated under the action of a constant internal pressure is considered. A rigid spherical particle is also suspended inside the liquid phase. Results in terms of bubble growth, particle dynamics, and liquid strain rate fields are reported and discussed for different geometrical and flow parameters.

\section{Mathematical model}

In Fig. 1, a (not to scale) schematic drawing of the computational domain is reported.

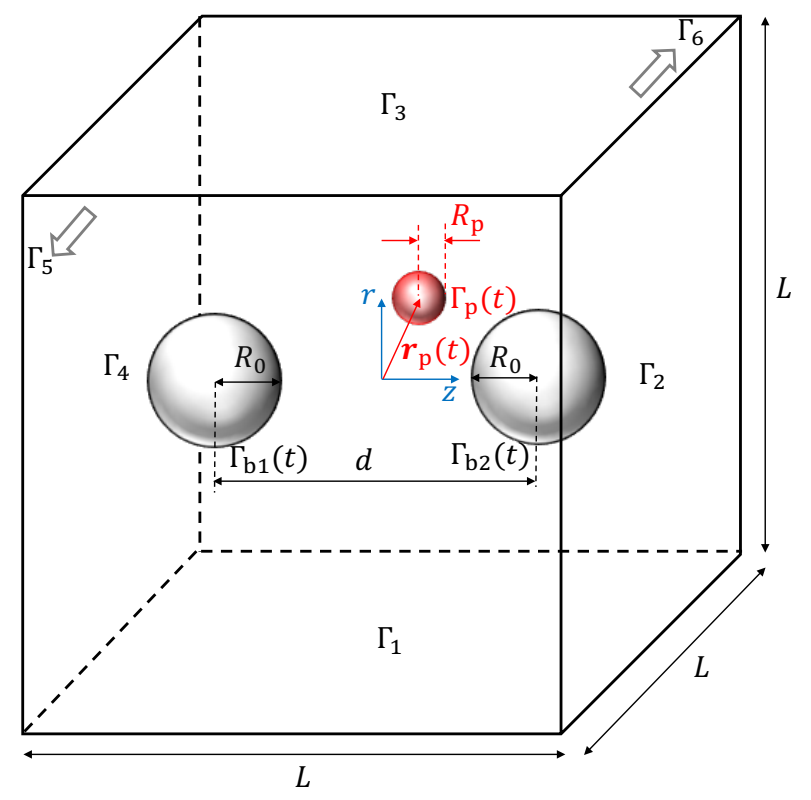

Figure 1: Sketch of the computational domain (not to scale).

A cubic Newtonian liquid domain with side $L$ is considered. A cylindrical system of coordinates is centered in the cube center with the positive $z$-direction oriented to the right. Two initially spherical gas bubbles with (equal) radius $R_{0}$ are situated with their centers on the $z$-axis at distance $d$ in a symmetric position with respect to the $r \theta$-plane at $z=0$. A rigid spherical particle with radius $R_{\mathrm{p}}$ is suspended in the liquid at an initial position $\boldsymbol{r}_{\mathrm{p}, 0}$ (with reference to the particle center). Due to the axisymmetry of the investigated system, the angular position of the particle is irrelevant. Thus, we initially set the particle at $\theta=0$, i.e., $\boldsymbol{r}_{\mathrm{p}, 0}=\left(z_{\mathrm{p}, 0}, r_{\mathrm{p}, 0}, 0\right)$. In what follows, we omit to specify the angular coordinate of the particle position.

For the sake of simplicity, we neglect the dif- 
fusion of the dissolved gas through the liquid and assume that the bubble growth is driven by a constant pressure difference between the bubbles and the liquid far from them. For this reason, only the liquid phase is mathematically described. Assuming the liquid phase to be incompressible and inertialess, its dynamics is governed by the mass and momentum balance equations in the Stokes formulation, reading

$$
\begin{aligned}
& \boldsymbol{\nabla} \cdot \boldsymbol{u}=0 \\
& \boldsymbol{\nabla} \cdot \boldsymbol{\tau}=\mathbf{0}
\end{aligned}
$$

where $\boldsymbol{u}$ is the velocity vector and $\boldsymbol{\tau}=$ $-p \boldsymbol{I}+2 \eta \boldsymbol{D}$ is the stress tensor, with $p$ the pressure, $\boldsymbol{I}$ the identity tensor, $\eta$ the Newtonian liquid viscosity, and $\boldsymbol{D}=\left(\boldsymbol{\nabla} \boldsymbol{u}+\boldsymbol{\nabla} \boldsymbol{u}^{\mathrm{T}}\right) / 2$ the symmetric part of the velocity gradient tensor.

The continuity and momentum balance equations in the liquid phase are solved with the following boundary conditions:

$$
\begin{gathered}
\boldsymbol{\tau} \cdot \boldsymbol{m}=-p_{\infty} \boldsymbol{m} \text { on } \Gamma_{1}-\Gamma_{6} \\
\boldsymbol{\tau} \cdot \boldsymbol{n}=\sigma \boldsymbol{n} \boldsymbol{\nabla} \cdot \boldsymbol{n}-p_{\mathrm{g}} \boldsymbol{n} \text { on } \Gamma_{\mathrm{b} 1}(t), \Gamma_{\mathrm{b} 2}(t) \\
\boldsymbol{u}=\boldsymbol{U}+\boldsymbol{\omega} \times \boldsymbol{r} \text { on } \Gamma_{\mathrm{p}}(t) \\
\boldsymbol{F}=\int_{\Gamma_{\mathrm{p}}(t)} \boldsymbol{\tau} \cdot \boldsymbol{n} d S \text { on } \Gamma_{\mathrm{p}}(t) \\
\boldsymbol{T}=\int_{\Gamma_{\mathrm{p}}(t)} \boldsymbol{r} \times(\boldsymbol{\tau} \cdot \boldsymbol{n}) d S \text { on } \Gamma_{\mathrm{p}}(t)
\end{gathered}
$$

Equation (4) expresses the outflow condition on the cubic domain boundaries, with $p_{\infty}$ the (constant) liquid pressure far from the bubbles and $\boldsymbol{m}$ the outwardly directed unit vector normal to boundaries $\Gamma_{1}-\Gamma_{6}$. Equation (5) is the Young-Laplace boundary condition on the moving interfaces $\Gamma_{\mathrm{b} 1}(t)$ and $\Gamma_{\mathrm{b} 2}(t)$ between the gas bubbles and the liquid, with $\sigma$ the surface tension, $p_{\mathrm{g}}$ the gas pressure, and $\boldsymbol{n}$ the unit vector normal to such interfaces and directed toward the liquid phase. It is worth mentioning that the pressure of the gas inducing bubble growth is assumed to be constant. Equation (6) is the rigid-body motion condition on the moving solid particle spherical surface $\Gamma_{\mathrm{p}}(t)$, where $\boldsymbol{r}$ denotes the position vector of a generic point on the particle surface, $\boldsymbol{U}$ and $\boldsymbol{\omega}$ are the particle translational and angular velocities (to be determined). Finally, Eqs. (7) and (8) specify the hydrodynamic force $\boldsymbol{F}$ and torque $\boldsymbol{T}$ acting on the particle. Since inertia is neglected, the particle is forceand torque-free. The absence of inertia also implies that no initial conditions are needed for the unknowns.

The balance equations and the boundary conditions given above are made dimensionless by choosing the bubbles' initial radius $R_{0}$ as the characteristic length, $\eta / \Delta p$, with $\Delta p=p_{\mathrm{g}}-p_{\infty}$, as the characteristic time, $R_{0} \Delta p / \eta$ as the characteristic velocity, and $\Delta p$ as the characteristic stress. Doing so, the capillary number $\mathrm{Ca}=R_{0} \Delta p /(2 \sigma)$ appears in Eq. (5). Moreover, three dimensionless geometrical parameters can be identified, i.e., the initial distance between the centers of the bubbles $d$, the particle radius $R_{\mathrm{p}}$, and the initial particle position $\boldsymbol{r}_{\mathrm{p}, 0}$. All the results 
presented in the following are given in terms of dimensionless quantities, the usual superscript ${ }^{*}$ being omitted for simplicity.

\section{Numerical technique and code vali- dation}

The equations constituting the mathematical model illustrated in Sec. 2 are solved through the Arbitrary Lagrangian Eulerian (ALE) Finite Element Method (FEM). The outcomes of our numerical simulations are the full velocity and stress fields dynamics in the whole liquid domain, as well as the dynamics of the gas bubbles and the solid particle. As reported above, the spherical particle is rigid, thus all its points have the same velocity and no stress is defined at its interior. Furthermore, the pressure making the bubbles grow is given as an input at the gas-liquid interface, so the velocity and stress fields in the gas phase are not solved. For these reasons, the computational domain is a cube of side $L$ with three initially spherical 'holes', i.e., the gas bubbles and the solid particle. The portion of space surrounding the bubbles and the particle is discretized by means of a mesh made of quadratic tetrahedra. On the solid-liquid and gas-liquid interfaces, the mesh aligns with element faces (quadratic triangles). We use quadratic $\left(P_{2}\right)$ interpolation for the velocity $\boldsymbol{u}$ and linear $\left(P_{1}\right)$ interpolation for the pressure $p$ (Taylor-Hood elements). In Fig. 2a, a detail of the computational mesh for a domain with two bubbles at initial distance $d=5.0$ and a particle with radius $R_{\mathrm{p}}=0.2$ initially placed at $\left(z_{\mathrm{p}, 0}, r_{\mathrm{p}, 0}\right)=(0,0.1)$ is shown. For clarity, only the surface mesh elements on the bubbles, the particle, and the cube faces $\Gamma_{5}$ and $\Gamma_{6}$ are shown.
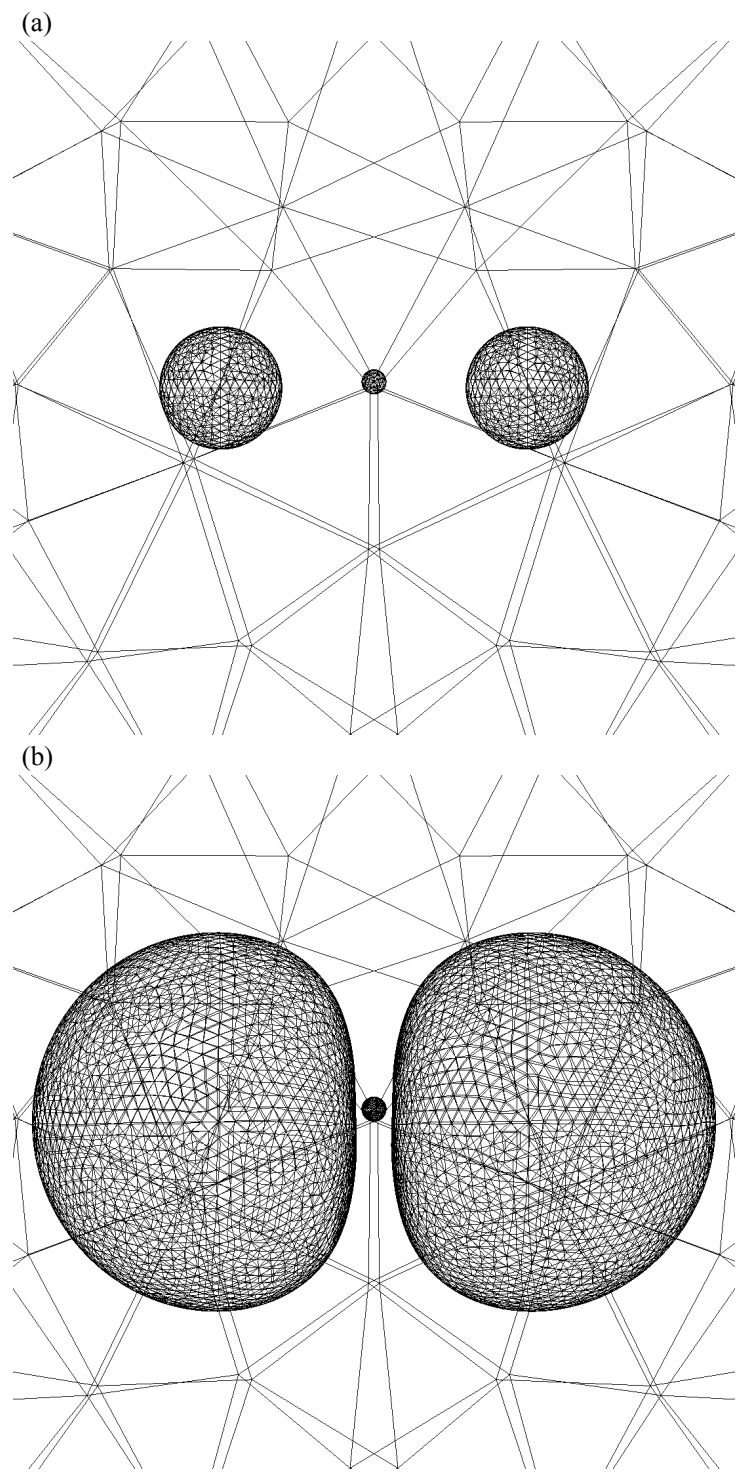

Figure 2: Zoom of the meshed computational domain for $d=5.0, R_{\mathrm{p}}=0.2,\left(z_{\mathrm{p}, 0}, r_{\mathrm{p}, 0}\right)=(0,0.1)$. (a) Initial configuration. (b) Configuration after bubble growth.

During the system evolution, the deformable interfaces between the gas inclusions 
and the liquid matrix need to be tracked: to do this, a FEM with second-order time discretization is defined on $\Gamma_{\mathrm{b} 1}$ and $\Gamma_{\mathrm{b} 2}$, where the normal component of the mesh velocity equals the normal component of the physical velocity, whereas the tangential velocity of the mesh nodes is such that the distribution of the elements on the interfaces is optimized. Compared to a Lagrangian one, this approach greatly reduces the distortion of the interface mesh. A detailed description of the adopted numerical procedure can be found in [28].

As bubbles inflate, approaching each other and squeezing the liquid in between them, which, in turn, transports the solid particle, the mesh volume elements progressively warp. Therefore, every time the mesh quality, in terms of the aspect of the 'worst' element in the domain, goes below a given threshold, a remeshing is performed [29, 30]. Moreover, since great deformations arise when the bubbles are close to each other, and possibly enclose the rigid particle, when necessary, mesh refinement is performed, ensuring the presence of a minimum number of volume elements in the gaps between the bubbles and between the bubbles and the particle. At every refinement step, each triangular mesh element on the solid-liquid and gas-liquid interfaces is divided in four, with the volume elements being accordingly rebuilt. With reference to the system whose initial computational mesh is displayed in Fig. 2a, we show in Fig. 2 $\mathrm{b}$ a detail of the mesh after bubbles have grown to a certain extent and the particle has slightly displaced in the positive $r$-direction. Since the bubbles have approached each other, nearly 'entrapping' the particle, both the bubbles and the particle surface meshes have been refined with respect to the initial resolution. It is worth remarking that, as all the sharpinterface methods, the technique used in the present work does not allow to deal with bubble coalescence and break-up. On the other hand, it allows to give a very accurate description of the thin layers in between the two bubbles and the bubbles and the particle, which is crucial with respect to the aim of this paper.

The side of the cubic computational domain is chosen sufficiently larger than the maximum dimension attained by the bubbles to ensure that the growth dynamics is unaffected by the outflow boundary conditions. In particular, $L=30.0$ satisfies this condition.

Preliminary simulations are performed to check space and time convergence, i.e., the initial mesh resolution and the time-step for the numerical solution of the equations given in Sec. 2 are chosen to ensure invariance of the results upon further refinements. This is achieved with meshes with 40 line elements on the bubbles' equators, 15 line elements on the particle equator, and 10 line elements on the cube sides, yielding a total initial number of tetrahedral volume elements around $2 \times 10^{4}$ (roughly doubled at the end of each simulation due to the above mentioned refinements) and time-steps in the order of $0.025 \eta / \Delta p$.

The simulations are performed on blades with two hexacore processors Intel Xeon E5649@2.53GHz and $48 \mathrm{~Gb}$ of RAM. For each set of parameters considered, the computational time needed to get the results presented in Sec. 4 is below 12 hours.

We validate our code by simulating the 




Figure 3: Comparison between the numerically computed temporal trend of the radius of a gas bubble in an infinite Newtonian liquid and the theoretical prediction given in Eq. (1) (see legend). The capillary number is $\mathrm{Ca}=1.111$.

spherical growth of a single gas bubble in an infinite Newtonian liquid and comparing our numerical bubble radius trend with the theoretical prediction from Favelukis et al. [9] given in Eq. (1). In Fig. 3, we plot the bubble normalized radius $R / R_{0}$ as function of the dimensionless time $t\left(p_{\mathrm{b}}-p_{\infty}\right) /(4 \eta)$ arising from our FEM simulations through blue circles and the prediction from Eq. (1) through a red solid line. The capillary number is $\mathrm{Ca}$ $=1.111$. An overlap of the two trends over the whole time window considered is found.

\section{Results}

As reported above, the balance equations given in Sec. 2 are solved through the ALE FEM to compute the velocity and pressure fields in the liquid matrix and the dynamics of the bubbles and the solid particle. We perform a parametric study with respect to the capillary number $\mathrm{Ca}$ and the geometrical parameters $d$ and $R_{\mathrm{p}}$. The capillary number is varied in the range [5.0,40.0]. The lower value is chosen slightly greater than $\mathrm{Ca}_{\min } \simeq 1$, which is the critical value at which the pressure force making the bubbles inflate is balanced by the surface tension force contrasting bubble inflation, namely, the value that discriminates between bubble contraction and growth. The higher value is estimated from operating conditions commonly used in foaming processes (see, e.g., Ref. [27]). The initial bubble-bubble distance $d$ is varied between 3.0, corresponding to an initial distance equal to the dimension of one bubble, and 7.0, where the hydrodynamic interactions between the gas inclusions are weak and the two bubbles behave almost as isolated. Finally, the particle radius $R_{\mathrm{p}}$ is varied between 0.1 and 0.3 in order to mimic situations of practical interest (again, see, for example, Ref. [27]).

In Fig. 4, we report the color maps of the strain rate $\dot{\epsilon}=\sqrt{2 \boldsymbol{D}: \boldsymbol{D}}$ computed on the $z r$-plane at different time values (see figure) during bubble growth. The capillary number $\mathrm{Ca}$ is equal to 5.0, the initial bubble distance is $d=5.0$, and the particle radius is $R_{\mathrm{p}}=0.2$. In Fig. 4 a there is no particle, whereas Figs. $4 \mathrm{~b}-\mathrm{d}$ refer to particle initial positions $\left(z_{\mathrm{p}, 0}, r_{\mathrm{p}, 0}\right)=(0,0.1),(0,1.0),(0,2.7)$, respectively (thus, in all the cases, the particle lies on the $r$-axis). When no particle is present between the bubbles (Fig. 4a), these initially grow with a spherical shape $(t=1.25,2.5)$, then, as hydrodynamic interactions start to be relevant, the bubbles preferentially grow on the side opposite to the other bubble, whereas 


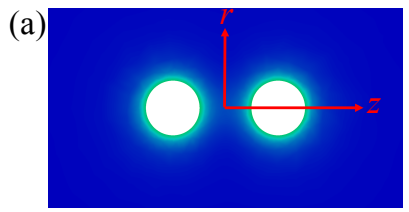

$t=1.25$



$t=2.5$

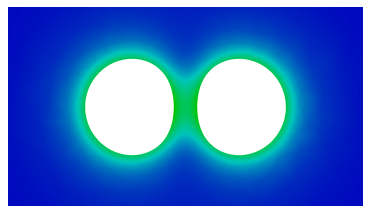

$t=3.75$



$t=5.0$



$t=6.25$

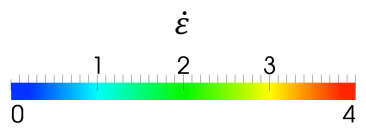

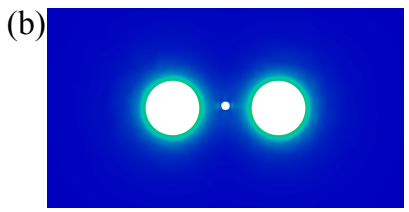

$t=1.25$

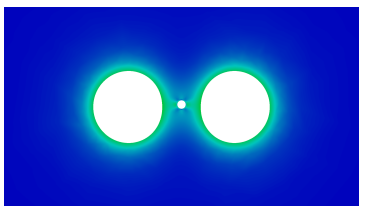

$t=2.5$

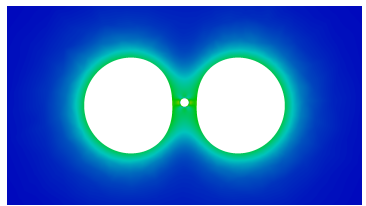

$t=3.75$

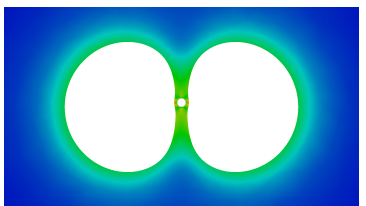

$t=5.0$



$t=6.25$

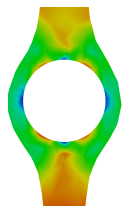



$t=1.25$

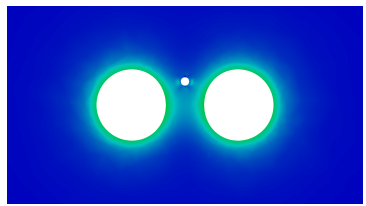

$t=2.5$



$t=3.75$

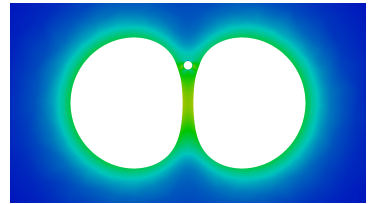

$t=5.0$



$t=6.25$





$t=1.25$

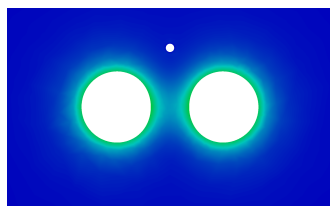

$t=2.5$

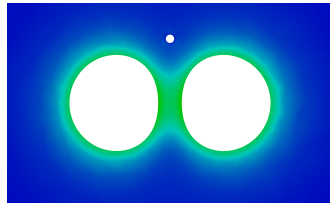

$t=3.75$



$t=5.0$

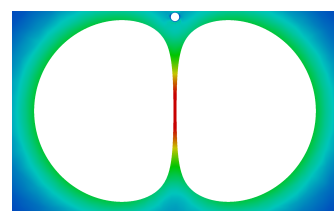

$t=6.25$

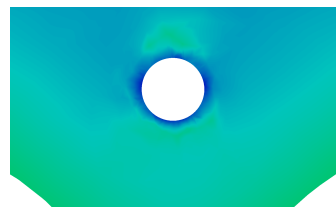

Figure 4: Color maps of the strain rate $\dot{\epsilon}=\sqrt{2 \boldsymbol{D}: \boldsymbol{D}}$ on the $z r$-plane for $\mathrm{Ca}=5.0$ and $d=5.0$ at 5 time instants (see figure) with no particle (a). $\dot{\epsilon}$-color maps on the $z r$-plane passing through the centers of the bubbles and the particle for $\mathrm{Ca}=5.0, d=5.0$, and $R_{\mathrm{p}}=0.2$ at 5 time instants (see figure) and $\left(z_{\mathrm{p}, 0}, r_{\mathrm{p}, 0}\right)=(0,0.1)(\mathrm{b}),(0,1.0)(\mathrm{c}),(0,2.7)(\mathrm{d})$. In b-d, a zoom of the portion of the $z r$-plane around the particle at the last time of the sequence is displayed at the bottom of the column.

the bubbles' sides 'facing' the other bubble in between them undergoes an almost biaxial become progressively flatter, and the liquid extensional flow, with the strain rate increas- 
ing as the liquid film separating the bubbles thins $(t=3.75,5.0,6.25)$. Therefore, even if the mathematical description of the bubble growth is simplified (see above), the dynamics of the phenomenon is the result of the interplay among bubble growth driving force, bubble-bubble hydrodynamic interaction, and viscous response of the thin liquid film between the gas inclusions. Figure $4 \mathrm{~b}$ shows a situation where the particle starts slightly above the line connecting the centers of the bubbles, i.e, the $z$-axis. The first snapshot $(t=1.25)$ shows that, until the bubbles are far enough from each other and from the particle, they retain a spherical shape during growth. However, the presence of the solid inclusion induces a shape alteration earlier than in the case with no particle $(t=2.5)$, with a strain rate enhancement in the liquid enclosed between the particle and the bubbles. As bubbles inflate, the particle moves in the positive $r$-direction due to the liquid extensional flow; however, the particle translational motion is much slower than the bubble growth, thus the particle gets 'entrapped' between the bubbles, inducing a curvature change in their boundaries, which conform to the particle shape $(t=5.0,6.25)$. At the bottom of Fig. $4 \mathrm{~b}$, a close view of the fluid domain around the particle at $t=6.25$ shows the curvature change of the bubbles (white regions surrounding the fluid) and the particle-induced disturbance in the strain rate field, with a structure of alternate $\dot{\epsilon}$-maxima and minima around the boundary of the solid phase. The occurrence of such strain rate extrema in the very thin layer between the bubbles and the particle results in stress conditions that may induce the rupture of the liquid film and influence the extent of the retraction of the liquid separating the bubbles, leading to different foam morphologies. The dynamics of a liquid film in the presence of a hole breaking its continuity goes beyond the scope of this work and is investigated elsewhere [31, 32. In Fig. 4 , snapshots of bubble growth are reported for $\left(z_{\mathrm{p}, 0}, r_{\mathrm{p}, 0}\right)=(0,1.0)$. At variance with the case in panel $b$, the particle has almost no effect on the bubble dynamics for a 'long' time (the morphology and strain rate in the first four snapshots in panel c are very similar to the corresponding ones in panel a). At the latest time $t=6.25$, the particle is surrounded by the bubbles, causing a curvature change and strain rate disturbance less pronounced as compared to the case reported in panel b (see also the zoom around the particle at the bottom of sequence c). Finally, in Fig. $4 \mathrm{~d}$, a sequence of images referring to $\left(z_{\mathrm{p}, 0}, r_{\mathrm{p}, 0}\right)=(0,2.7)$ is displayed. The bubble growth is substantially unaffected by the presence of the particle during the whole dynamics (compare with morphology and color maps in Fig. 4a). The particle, in turn, 'escapes' the bubbles due to the liquid extensional flow: at $t=6.25$, when the bubbles are almost coalescing, the particle has been transported in the bulk of the liquid above the bubbles, as also shown in the close view at the bottom of column d. In view of the cases just presented, it can be inferred that the physical mechanism for particle capture/escape is based on the competition of bubble growth and the extensional flow induced by bubble growth in between the bubbles, resulting in a different particle fate depending on the initial position 
of the solid inclusion.

In Fig. 5, the $\dot{\epsilon}$-color maps on the $z r$ plane passing through the centers of the bubbles and the particle are reported for the same parameters considered above $(\mathrm{Ca}=5.0$, $\left.d=5.0, R_{\mathrm{p}}=0.2\right)$ and for $\left(z_{\mathrm{p}, 0}, r_{\mathrm{p}, 0}\right)=$ $(0.5,0.1),(0.5,1.0)$, namely, two initial positions in which the particle starts closer to the bubble on the right (of course, since the bubble are equal and are inflated with the same law, a symmetric scenario would occur if the particle was closer to the left bubble). As expected, the particle affects the growth of the closer bubble earlier than the other, inducing a curvature change in the bubble profile (for example, see the second snapshot in Fig. 5a). The closer bubble, in turn, pushes the particle in the negative $z$-direction and, due to the extensional flow field, towards the positive $r$-direction. For the particle initial position considered in Fig. 5a, the bubbles entrap the particle. The zoom at the bottom of Fig. 4 a shows that, at the end of bubble growth, the thickness of the liquid film around the solid inclusion is thinner on one side, and the strain rate has a minimum there. In Fig. $5 \mathrm{~b}$, snapshots of bubble growth are reported for $\left(z_{\mathrm{p}, 0}, r_{\mathrm{p}, 0}\right)=(0.5,1.0)$. At variance with the case considered in panel a, here the particle has little effect even on the closer bubble for a long time. At the latest time considered $(t=6.25)$, the particle gets surrounded by the bubbles near their edge in the $r$-direction, causing a slight curvature change in the closer bubble and a moderate strain rate disturbance in the liquid, as also highlighted by the zoom at the bottom of the sequence.

Besides morphological information, the se-



$t=1.25$

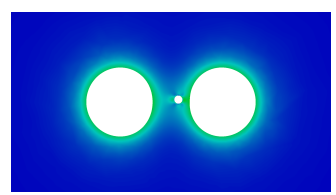

$t=2.5$

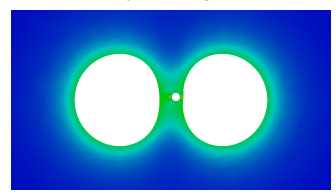

$t=3.75$

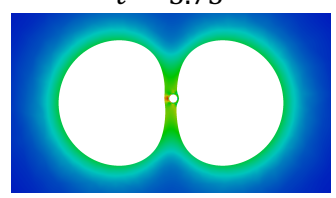

$t=5.0$



$t=6.25$

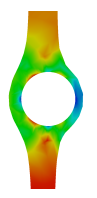

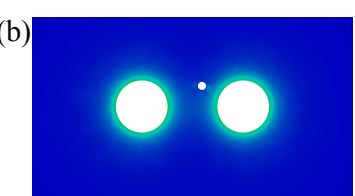

$t=1.25$

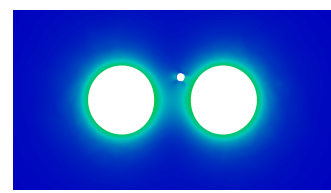

$t=2.5$

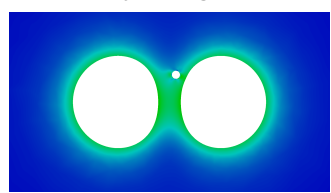

$t=3.75$

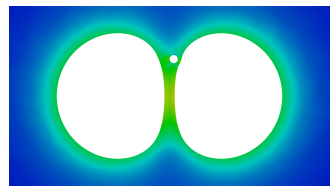

$t=5.0$

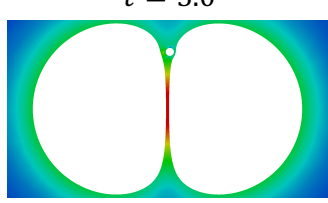

$t=6.25$

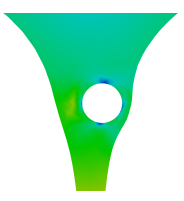

$\dot{\varepsilon}$



Figure 5: Color maps of the strain rate $\dot{\epsilon}=\sqrt{2 \boldsymbol{D}: \boldsymbol{D}}$ on the $z r$-plane passing through the centers of the bubbles and the particle for $\mathrm{Ca}=5.0, d=5.0$, and $R_{\mathrm{p}}=0.2$ at 5 time instants (see figure) and $\left(z_{\mathrm{p}, 0}, r_{\mathrm{p}, 0}\right)=(0.5,0.1)(\mathrm{a}),(0.5,1.0)(\mathrm{b})$. A zoom of the portion of the $z r$-plane around the particle at the last time of the sequence is displayed at the bottom of each column. 


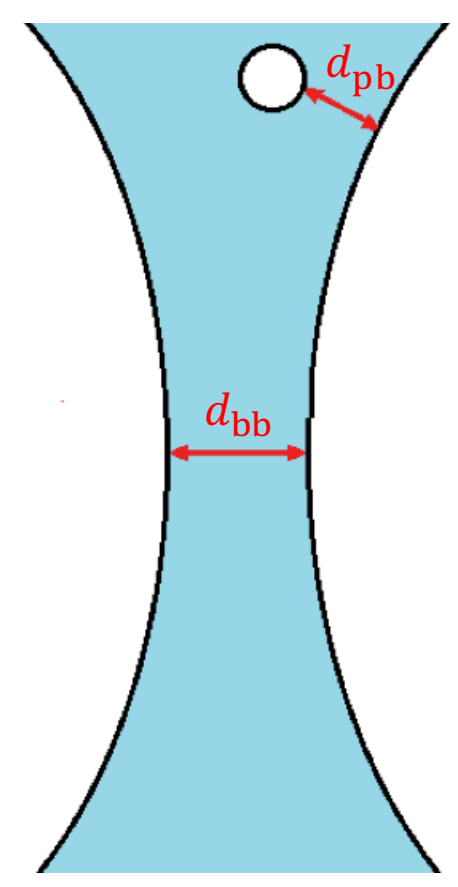

Figure 6: Sketch to illustrate the particle capture criterion.

quences of snapshots in Figs. 4 and 5 show that, for a specific set of physical and geometrical parameters $\mathrm{Ca}, d$, and $R_{\mathrm{p}}$, the particle can be driven out of the liquid film between the bubbles or be entrapped between them at the end of bubble growth (i.e., before coalescence occurs) depending on its initial position. In situations like the ones displayed in Figs. 4 and 5, one can discriminate 'by eye' which of the two scenarios occurs. A more quantitative and systematic criterion is needed in the general case. The criterion adopted in this work is based on the comparison of the particle-bubble and bubble-bubble distances. A sketch aimed at illustrating such criterion is reported in Fig. 6; at the end of each simulation, we compute the ratio between the minimum bubble-bubble distance $d_{\mathrm{bb}}$ and the minimum particle-bubble distance $d_{\mathrm{pb}}$; if this ratio is higher than 1 , the particle is considered to get enclosed by the bubbles, otherwise it is considered to escape the bubbles. In summary, given the physical and geometrical parameters, for every $z_{\mathrm{p}, 0}$, a 'critical' $r_{\mathrm{p}, 0}$ exists below which the particle gets entrapped by the bubbles and above which it escapes them. For each set of $\mathrm{Ca}, d$, and $R_{\mathrm{p}}$, we run several simulations by changing the initial particle position in the liquid volume surrounding the bubbles, also considering the zone near a bubble surface far from the other bubble, e.g., the region to the right of the right bubble. Of course, particles initially released in this region cannot be entrapped between the two bubbles. However, they can achieve a very small distance with the closest bubble before bubble coalescence (i.e., $d_{\mathrm{pb}}<d_{\mathrm{bb}}$ ), leading to an high strain-rate region that, in turn, can anticipate bubble rupture as compared with the case where no particle is present. In what follows, we refer to this situation as 'particle captured by the bubble' that is a more general case of 'particle entrapped between two bubbles'.

In Fig. 7k, we plot the critical initial radial coordinate $r_{\mathrm{c}}$ as function of the particle initial axial coordinate $z_{\mathrm{p}, 0}$ for the same capillary number and initial bubble distance of Figs. 4 and $5(\mathrm{Ca}=5.0, d=5.0)$ and three different values of the particle radius, i.e., $R_{\mathrm{p}}=0.1,0.2,0.3$ (see legend for the symbols). The circular arc represents the initial shape of the right bubble. Due to symmetry, only the portion of the $z r$-plane with positive $z$ is shown, the plots in the portion with 

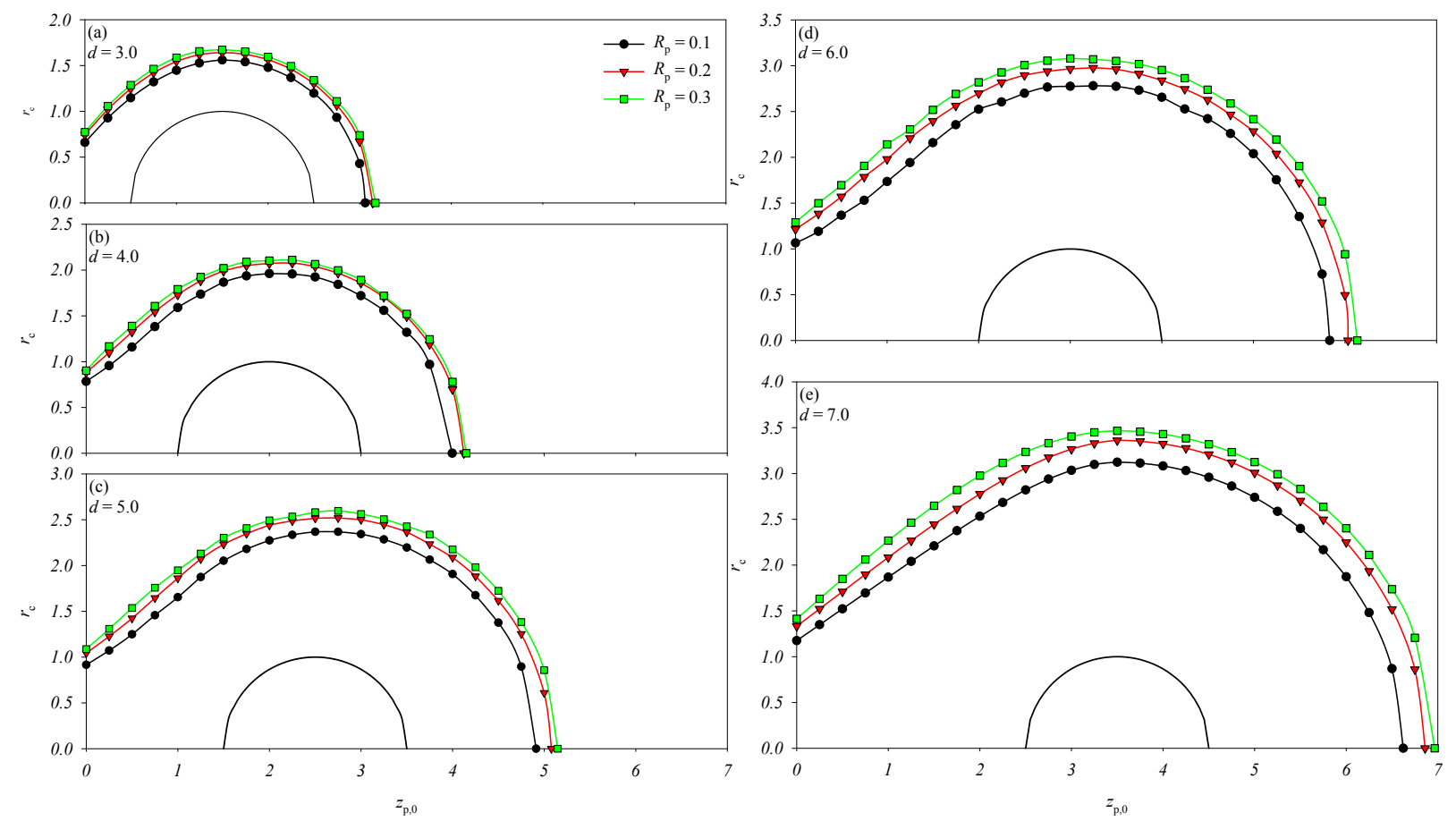

Figure 7: Particle critical initial radial position $r_{\mathrm{c}}$ as function of its initial axial position $z_{\mathrm{p}, 0}$ at $\mathrm{Ca}=5.0$ and $d=3.0$ (a), 4.0 (b), $5.0(\mathrm{c}), 6.0$ (d), 7.0 (e). In each panel, three values of the particle radius are considered, i.e., $R_{\mathrm{p}}=0.1,0.2,0.3$ (see legend). The solid circular arc represents the initial shape of the right bubble.

negative $z$ being obtainable by mirroring the data with respect to the $r$-axis. For each $R_{\mathrm{p}^{-}}$ value, the $r_{\mathrm{c}}$-curve divides the $z r$-plane into two regions: the one below the curve identifies the starting positions for which the particle is captured by the closest bubble or remains entrapped between the two bubbles, while the one above the curve identifies the starting positions for which the particle escapes the bubbles. For all the $R_{\mathrm{p}}$-values considered, the same qualitative $r_{\mathrm{c}}$-trend emerges: moving from $z=0$ in the positive $z$-direction, $r_{\mathrm{c}}$ first increases, namely, the attraction basin of particle capture enlarges. Therefore, a parti- cle starting equidistant from the two bubbles (i.e., on the $r$-axis) is the less likely to be entrapped between them, since at $z=0$ the entrapment attraction basin is the narrowest. By further increasing $z_{\mathrm{p}, 0}$, the $r_{\mathrm{c}}$-curve almost 'conforms' to the bubble circular initial profile. Indeed, when the particle starts in this region, the other bubble has a negligible effect on its dynamics and the particle, depending on its initial distance, is incorporated by the closest bubble or escapes it. The $R_{\mathrm{p}}$-value has no qualitative effect on such behavior, yet a quantitative effect is apparent: at increasing $R_{\mathrm{p}}$, the $r_{\mathrm{c}}$-trend moves upwards. Actually, 
this result could be expected since bigger particles are more easily captured by the growing bubbles (or the single growing bubble).

In Figs. $7 \mathrm{a}-\mathrm{b}$ and $7 \mathrm{~d}$-e, smaller and larger initial bubble distances are considered, i.e., $d=3.0,4.0$ and $d=6.0,7.0$, respectively. The trends observed for $d=5.0$ (panel a) still hold at varying the bubble distance. Focusing on the region in the proximity of $z=0$, namely, when the particle is equidistant from the two bubbles or slightly closer to one of them, the comparison among panels a-e shows that (at any given $R_{\mathrm{p}}$-value) the amplitude of the capture attraction basin enlarges with $d$. This could appear counterintuitive, since one could expect that a particle more easily escapes bubbles initially further from each other. However, it should be considered that, as the bubbles grow under the action of a constant inflation pressure, initially further bubbles can grow more before coalescence, thus entrapping particles at larger $r$-coordinates. For what concerns the effect of $R_{\mathrm{p}}$, like at $d=5.0$, the entrapment attraction basin is larger at increasing $R_{\mathrm{p}}$.

In order to study the effect of the capillary number on particle capture, in Fig. 8 we plot $r_{\mathrm{c}}\left(z_{\mathrm{p}, 0}\right)$ for $d=5.0, R_{\mathrm{p}}=0.2$, and four Ca-values, i.e., $\mathrm{Ca}=5.0,10.0,20.0$ and 40.0. As expected, by increasing $\mathrm{Ca}$, namely, by increasing the inflation pressure or decreasing the surface tension contrasting bubble growth, the particle entrapment attraction basin enlarges. On the other hand, it is apparent from Fig. 8 that such enlargement seems to asymptotize with Ca.

The results obtained by direct numerical simulations can be compared with a very sim-

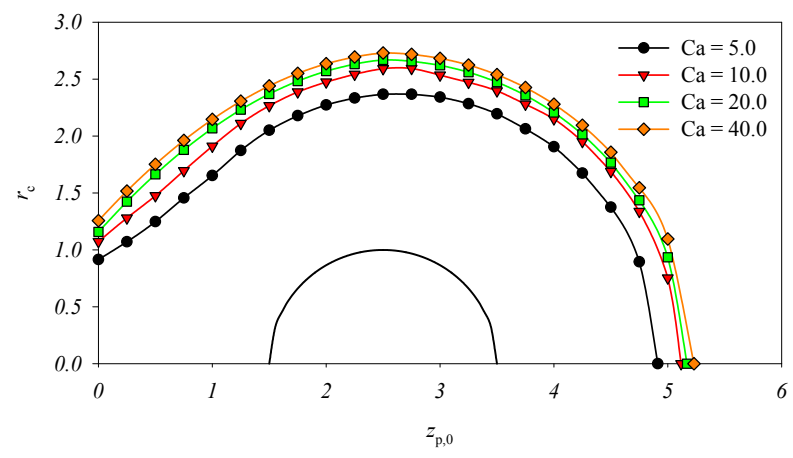

Figure 8: Particle critical initial radial position $r_{\mathrm{c}}$ as function of its initial axial position $z_{\mathrm{p}, 0}$ at $d=5.0$, $R_{\mathrm{p}}=0.2$, and $\mathrm{Ca}=5.0,10.0,20.0,40.0$ (see legend). The solid circular arc represents the initial shape of the right bubble.

plified theoretical prediction based on the single bubble spherical growth given by Eq. (1). The mass conservation law implies that the velocity of the fluid in a generic radial position $r$ with respect to the center of the bubble is given by $v(r, t)=\dot{R}(t) R^{2}(t) / r^{2}$, where $R(t)$ is the position of the bubble moving boundary, whose evolution is reported in Eq. (1). (All the quantities are made dimensionless as explained in Sec. 2.) Assuming that the particle moves as a tracer, i.e., according to the velocity field induced by the inflating bubble in the surrounding fluid, its translational motion can be readily obtained by integrating the kinematic equation $d r_{\mathrm{p}} / d t=v\left(r_{\mathrm{p}}, t\right)$ and reads

$$
\begin{aligned}
& r_{\mathrm{p}}(t)= \\
& \frac{1}{\mathrm{Ca}}\left(\mathrm{Ca}^{3}\left(r_{\mathrm{p}, 0}^{3}-1\right)+\left[(\mathrm{Ca}-1) e^{\frac{t}{4}}+1\right]^{3}\right)^{1 / 3}
\end{aligned}
$$


with $r_{\mathrm{p}, 0}$ the initial particle position. To compare the numerical results shown above and the analytical predictions, we assume that the two bubbles grow keeping the spherical shape. Hence, the maximum radius to which the bubble is allowed to grow is $d / 2$. For any $\mathrm{Ca}, R_{\mathrm{p}}$, and $d$, it is then possible to evaluate the critical value of the particle initial position $r_{\mathrm{p}, 0}$ such that the bubble 'touches' the particle boundary at the same time at which it touches the other bubble, namely, when $r_{\mathrm{p}}(t)-d / 2=R_{\mathrm{p}}$. For instance, by choosing $\mathrm{Ca}=5.0, d=5.0$ and $R_{\mathrm{p}}=0.1$, the critical initial particle position is equal to 1.435 , which is lower than the values arising from numerical simulations in the same conditions (see Fig. 7k, where the distance of the black points from the center of the bubble is wherever greater than 2). Analogous results can be obtained at varying the parameters. Hence, the simplified analytical argument underestimates the extent of the capture attraction basin as compared to the numerical results, suggesting that bubble-bubble and bubble-particle hydrodynamic interactions are important for the investigated system.

\section{Conclusions}

In this paper, the growth and impingement dynamics of two gas bubbles in a Newtonian liquid in the presence of a solid spherical particle is studied by means of 3D Arbitrary Lagrangian Eulerian (ALE) Finite Element Method (FEM) numerical simulations.

As soon as, during their growth, the two initially spherical bubbles start to 'feel' each other, they lose their spherical shape, with the side facing the other bubble becoming almost flat. An essentially biaxial extensional flow takes place in the thinning liquid layer between the gas inclusions. Then, depending on its initial position relatively to the bubbles, the solid particle can be 'captured' by the bubbles or 'escape' them. The physical (e.g., bubble pressure, surface tension) and geometrical (e.g, particle size, bubble distance) parameters quantitatively influence the extensions of the entrapment and escape attraction basins.

Although some simplifications are introduced in the simulations (only two bubbles with the same initial dimension are considered and both grow under the action of a constant pressure difference with respect to the surrounding liquid), the results presented in this paper widen the comprehension of the growth and impingement dynamics of gas bubbles in the presence of solid impurities, which could find application in foam processing. Indeed, if during foaming a solid particle gets enclosed in the thinning film between two growing bubbles, it could induce the rupture of such film, and consequently influence the morphology of the resulting material.

Future developments will include an investigation of the phenomenon in viscoelastic matrices.

\section{References}

[1] H. E. Naguib, C. B. Park, U. Panzer, N. Reichelt, Strategies for achieving ultra low-density polypropylene foams, Polymer Engineering \& Science 42 (7) (2002) 1481-1492. 
[2] S. T. Lee, C. B. Park, Foam extrusion: principles and practice, CRC press, 2014.

[3] L. Rayleigh, On the pressure developed in a liquid during the collapse of a spherical cavity, The London, Edinburgh, and Dublin Philosophical Magazine and Journal of Science 34 (1917) 94-98.

[4] H. Lamb, The early stages of a submarine explosion, The London, Edinburgh, and Dublin Philosophical Magazine and Journal of Science 45 (266) (1923) 257265.

[5] N. Ramesh, D. H. Rasmussen, G. A. Campbell, The heterogeneous nucleation of microcellular foams assisted by the survival of microvoids in polymers containing low glass transition particles. part i: Mathematical modeling and numerical simulation, Polymer Engineering \& Science Science 34 (22) (1994) 1685-1697.

[6] J. J. Feng, C. A. Bertelo, Prediction of bubble growth and size distribution in polymer foaming based on a new heterogeneous nucleation model, Journal of Rheology 48 (2) (2004) 439-462.

[7] P. S. Epstein, M. S. Plesset, On the stability of gas bubbles in liquid-gas solutions, The Journal of Chemical Physics 18 (11) (1950) 1505-1509.

[8] L. Trilling, The collapse and rebound of a gas bubble, Journal of Applied Physics 23 (1) (1952) 14-17.
[9] M. Favelukis, R. J. Albalak, Bubble growth in viscous newtonian and nonnewtonian liquids, The Chemical Engineering Journal and The Biochemical Engineering Journal 63 (3) (1996) 149-155.

[10] O. Busaryev, T. K. Dey, H. Wang, Z. Ren, Animating bubble interactions in a liquid foam, ACM Transactions on Graphics (TOG) 31 (4) (2012) 63.

[11] R. J. Pugh, Bubble and foam chemistry, Cambridge University Press, 2016.

[12] M. A. Shafi, J. G. Lee, R. W. Flumerfelt, Prediction of cellular structure in free expansion polymer foam processing, Polymer Engineering \& Science 36 (14) (1996) 1950-1959.

[13] M. Shimoda, I. Tsujimura, M. Tanigaki, M. Ohshima, Polymeric foaming simulation for extrusion processes, Journal of Cellular Plastics 37 (6) (2001) 517-536.

[14] S. Everitt, O. Harlen, H. Wilson, Bubble growth in a two-dimensional viscoelastic foam, Journal of non-Newtonian fluid mechanics 137 (1) (2006) 46-59.

[15] S. N. Leung, C. B. Park, D. Xu, H. Li, R. G. Fenton, Computer simulation of bubble-growth phenomena in foaming, Industrial \& engineering chemistry research 45 (23) (2006) 7823-7831.

[16] I. Tsivintzelis, A. G. Angelopoulou, C. Panayiotou, Foaming of polymers with supercritical co2: an experimental and 
theoretical study, Polymer 48 (20) (2007) 5928-5939.

[17] K. Taki, Experimental and numerical studies on the effects of pressure release rate on number density of bubbles and bubble growth in a polymeric foaming process, Chemical Engineering Science 63 (14) (2008) 3643-3653.

[18] A. Sharma, E. Ruckenstein, An analytical nonlinear theory of thin film rupture and its application to wetting films, Journal of colloid and interface science $113(2)$ (1986) 456-479.

[19] P. C. Lee, J. Wang, C. B. Park, Extruded open-cell foams using two semicrystalline polymers with different crystallization temperatures, Industrial \& engineering chemistry research 45 (1) (2006) 175-181.

[20] G. C. Frye, J. C. Berg, Antifoam action by solid particles, Journal of colloid and interface science 127 (1) (1989) 222-238.

[21] X. Zhang, H. Davis, C. Macosko, A new cell opening mechanism in flexible polyurethane foam, Journal of cellular plastics 35 (5) (1999) 458-476.

[22] G. Harikrishnan, T. U. Patro, D. Khakhar, Polyurethane foamclay nanocomposites: nanoclays as cell openers, Industrial \& engineering chemistry research $45 \quad$ (21) (2006) 7126-7134.

[23] G. Taylor, The dynamics of thin sheets of fluid. iii. disintegration of fluid sheets,
Proceedings of the Royal Society of London A: Mathematical, Physical and Engineering Sciences 253 (1274) (1959) 313321.

[24] F. Culick, Comments on a ruptured soap film, Journal of applied physics 31 (6) (1960) 1128-1129.

[25] G. Debrégeas, P. G. De Gennes, F. Brochard-Wyart, The life and death of" bare" viscous bubbles, Science 279 (5357) (1998) 1704-1707.

[26] E. Sabadini, R. F. Ungarato, P. B. Miranda, The elasticity of soap bubbles containing wormlike micelles, Langmuir 30 (3) (2014) 727-732.

[27] D. Tammaro, G. D’Avino, E. Di Maio, R. Pasquino, M. M. Villone, D. Gonzales, M. Groombridge, N. Grizzuti, P. L. Maffettone, Validated modeling of bubble growth, impingement and retraction to predict cell-opening in thermoplastic foaming, Chemical Engineering Journal 287 (2016) 492-502.

[28] M. M. Villone, M. A. Hulsen, P. D. Anderson, P. L. Maffettone, Simulations of deformable systems in fluids under shear flow using an arbitrary lagrangian eulerian technique, Computers \& Fluids 90 (2014) 88-100.

[29] H. H. Hu, N. A. Patankar, M. Y. Zhu, Direct numerical simulations of fluid-solid systems using the arbitrary lagrangianeulerian technique, Journal of Computational Physics 169 (2) (2001) 427-462. 
[30] N. O. Jaensson, M. A. Hulsen, P. D. Anderson, Stokes-cahn-hilliard formulations and simulations of two-phase flows with suspended rigid particles, Computers \& Fluids 111 (2015) 1-17.

[31] N. Savva, J. W. Bush, Viscous sheet retraction, J. Fluid Mech. 626 (2009) 211240.

[32] M. M. Villone, G. D'Avino, E. Di Maio, M. A. Hulsen, P. L. Maffettone, Modeling and simulation of viscoelastic film retraction, J. Non-Newtonian Fluid Mech. 249 (2017) 26-35. 